\title{
Protein Kinase CK2 Impairs Spatial Memory Formation through Differential Cross Talk with PI-3 Kinase Signaling: Activation of Akt and Inactivation of SGK1
}

\author{
Chih C. Chao, ${ }^{1,2}$ Yun L. Ma, ${ }^{1}$ and Eminy H. Y. Lee ${ }^{1}$ \\ ${ }^{1}$ Institute of Biomedical Sciences, Academia Sinica, Taipei 115, Taiwan, and ${ }^{2}$ Institute of Neuroscience, Tzu-Chi University, Hualien 970 , Taiwan
}

\begin{abstract}
Casein kinase II (CK2) is a multifunctional serine/threonine protein kinase that is associated with the development of neuritogenesis and synaptic plasticity. The phosphoinositide 3-kinase (PI-3K)/Akt pathway is implicated in long-term memory formation. In addition, serum- and glucocorticoid-inducible kinase 1 (SGK1) is another downstream target of PI-3K signaling that was shown to play an important role in spatial memory formation. Whether CK2 may also affect memory formation and whether CK2 interacts with Akt and SGK1 during this process is unknown. In the present study, we found that water maze training significantly decreased CK2 activity in the rat hippocampal CA1 area but not in the dentate gyrus (DG) area. Transfection of the dominant negative mutant of CK2, CK2 $\alpha \mathrm{A}^{156}$, to the CA1 area, but not to the DG area, decreased CK2 activity but enhanced spatial memory formation. Meanwhile, it increased SGK1 phosphorylation at Ser422, decreased Akt phosphorylation at Ser473, and increased cAMP response element-binding protein phosphorylation at Ser133. Transfection of the constitutively active SGK1, SGKS422D, enhanced whereas transfection of the wild-type Akt impaired spatial memory formation. Also, administration of the protein phosphatase $2 \mathrm{~A}$ inhibitor, fostriecin, reversed the memory-impairing effect of CK $2 \alpha$ WT. It also reversed the effect of CK2 $\alpha$ WT in decreasing SGK1 phosphorylation. Akt Ser 473 phosphorylation was moderately increased by CK2 $\alpha \mathrm{WT}$ and fostriecin treatment, but AktS473A mutant transfection reversed the memory-impairing effect of CK2 $\alpha$ WT. These results together suggest that CK2 impairs spatial memory formation through differential cross talk with PI-3 kinase signaling by activation of Akt and inactivation of SGK1 through protein phosphatase 2A.
\end{abstract}

Key words: protein kinase CK2; serum- and glucocorticoid-inducible kinase 1; Akt; protein phosphatase 2A; spatial memory formation; hippocampus

\section{Introduction}

Casein kinase II (CK2) is a multifunctional and ubiquitous serine/threonine protein kinase (Pinna and Meggio, 1997; Litchfield 2003). The CK2 holoenzyme consists of $\alpha, \alpha^{\prime}$, (catalytic) and $\beta$ (regulatory) subunits, which associate to form $\alpha 2 \beta 2, \alpha^{\prime} 2 \beta 2$, and $\alpha \alpha^{\prime} \beta 2$ heterotetramers (Chester et al., 1995). The highest CK2 activity was found in the brain and testis of adult rats $(\mathrm{Na}-$ kajo et al., 1986). There are $>300$ substrates for protein kinase CK2 that are involved in signal transduction, cytoskeleton structure, cell-cell adhesion, and gene expression (Meggio and Pinna, 2003). In addition, CK2 was found to mediate the neurotrophic response of brain-derived neurotrophic factor in hippocampal slices (Blanquet, 2002) and the neurotrophic effect of glial cell line-derived neurotrophic factor on dopamine neurons (Chao et al., 2006). Furthermore, CK2 is associated with the development of neuritogenesis and synaptic plasticity. For example, decrease in CK2 activity inhibits microtubule assembly that is required for

Received Jan. 19, 2007; revised May 1, 2007; accepted May 1, 2007

This work was supported by National Science Council of Taiwan Grant NSC 95-2321-B-320-001 and by a Research Fund from the Institute of Biomedical Sciences, Academia Sinica, Taiwan.

Correspondence should be addressed to Dr. Eminy H. Y. Lee, Institute of Biomedical Sciences, Academia Sinica, Taipei 115, Taiwan. E-mail: eminy@gate.sinica.edu.tw.

D0I:10.1523/JNEUROSCI.1531-07.2007

Copyright $\odot 2007$ Society for Neuroscience $\quad$ 0270-6474/07/276243-06\$15.00/0 promoting neuritogenesis (Ulloa et al., 1993). CK2 activity is increased after the induction of long-term potentiation (LTP) in the rat hippocampus (Charriaut-Marlangue et al., 1991).

The phosphoinositide 3-kinase (PI-3K)/Akt pathway is implicated in LTP and consolidation of auditory fear memory and recognition memory (Lin et al., 2001; Horwood et al., 2006). In addition, CK2 is known to phosphorylate Akt, and CK2 upregulation of Akt activity mediates the anti-apoptotic effect of CK2 in Jurkat cells (Di Maira et al., 2005). Although CK2 activation was found to be associated with LTP (Charriaut-Marlangue et al., 1991), whether CK2 may also affect memory processing through interaction with Akt is not known. Serum- and glucocorticoidinducible kinase (SGK) is another downstream target of PI-3KPDK1 (phosphoinositide-dependent protein kinase-1) signaling (Kobayashi and Cohen, 1999). SGK1 has recently been demonstrated to play an important role in spatial memory formation (Tsai et al., 2002; Lee et al., 2006). A higher SGK mRNA level was found in fast-learning rats than slow-learning rats in the water maze learning task. Furthermore, overexpression of SGK1 in the hippocampal CA1 area improves, whereas expression of SGKS422A, the dominant negative mutant of SGK, impairs spatial memory formation (Tsai et al., 2002). But whether CK2 may interact with SGK1 during memory processing is also unknown. Based on the above findings, we hypothesized that CK2 activation 
in the hippocampus would facilitate spatial memory formation, and this is probably mediated through CK2 signaling to Akt and SGK1. The present study examined this hypothesis.

\section{Materials and Methods}

Animals. Adult male Sprague Dawley rats $(250-350 \mathrm{~g})$ bred in the Institution of Biomedical Sciences, Academia Sinica, were used. They were maintained on a $12 \mathrm{~h}$ light/dark cycle with food and water available continuously. For DNA transfection and drug administration, rats were subjected to stereotaxic surgery as described in supplemental material (available at www.jneurosci.org). Transfection and infusion were made at a rate of $0.1 \mu \mathrm{l} / \mathrm{min}$, and $0.5 \mu \mathrm{l}$ was delivered to each side of the hippocampus. Animals were subjected to water maze learning $48 \mathrm{~h}$ after DNA transfection or killed at different time points after drug infusion.

Plasmid DNA construction. Plasmid DNA was constructed as described previously (Chao et al., 2006; Lee et al., 2006). The sgk gene has different isoforms, and here we examined $s g k 1$. The full-length reverse transcription PCR products of the rat brain $C K 2 \alpha, s g k 1$, and akt genes were inserted in frame into the pcDNA3 vector with a hemagglutinin (HA) tag, and their sequences were verified by DNA sequencing. Mutant plasmid CK2 $\alpha \mathrm{A}^{156}$, SGKS422A, SGKS422D, and AktS473A were generated by site-directed mutagenesis. Branched polyethylenimine ( $25 \mathrm{kDa}$; Aldrich, Milwaukee, WI) was used as the transfection agent (Abdallah et al., 1996), and the equivalence of nitrogen per DNA phosphate was 10 for SGK1 and Akt and six for CK2 $\alpha$.

Plasmid DNA and drug injection. To assess the effects of various plasmid DNA transfections on spatial memory formation, animals were divided into different groups to receive $1.0 \mu \mathrm{g}$ of pcDNA3-HA or various plasmid DNA transfections as described below.

To study the interaction between CK2 $\alpha$ and protein phosphatase $2 \mathrm{~A}$ (PP2A) on SGK1 and Akt phosphorylation, animals were divided into three groups to receive pcDNA3-HA transfection $(1.0 \mu \mathrm{g})$ plus PBS infusion $(0.5 \mu \mathrm{l}), \mathrm{CK} 2 \alpha \mathrm{WT}$ transfection plus PBS infusion, and CK2 $\alpha \mathrm{WT}$ transfection plus fostriecin (FST) infusion (15 pg). Detailed procedures are given in supplemental material (available at www.jneurosci.org).

To examine the interaction between CK $2 \alpha$ and Akt on spatial memory formation, animals were divided into three groups to receive pcDNA3-HA transfection twice (1.0 $\mu \mathrm{g}$ each), pcDNA3-HA plus $\mathrm{CK} 2 \alpha \mathrm{WT}$ transfection, and AktS473A plus CK2 $\alpha \mathrm{WT}$ transfection. The interval between the two transfections was $1 \mathrm{~h}$. Water maze learning started $48 \mathrm{~h}$ after the second DNA transfection.

CK2 activity assay. CK2 activity assay was described previously (Chao et al., 2006) and detailed in supplemental material (available at www. jneurosci.org). Briefly, CK2 activity was measured in $5 \mu \mathrm{g}$ protein aliquots from each sample by using the protein kinase CK2 assay kit (Millipore, Bedford, MA). The synthetic peptide and $\left[\gamma_{-}{ }^{32} \mathrm{P}\right]$-ATP were used as the substrates, and a kinase inhibitor was added to block the activity of other serine/threonine kinases. Total CK2 activity was calculated by subtracting the blank (without the peptide substrate) from the ${ }^{32} \mathrm{P}$ radioactivity incorporated in the presence of the substrate.

Western blot. Fifteen micrograms of protein extract was subjected to SDS-PAGE and transferred to the polyvinylidene difluoride membrane. The membrane was first incubated with anti-p422SGK (1:500; Santa Cruz Biotechnology, Santa Cruz, CA), anti-SGK1 (1:1000; Millipore), anti-p473Akt (1:500; Cell Signaling Technology, Beverly, MA), anti-Akt (1:500; Cell Signaling Technology), anti-p133CREB (1:400; Cell Signaling Technology), anti-CREB (1:1000; Millipore), or anti- $\beta$-actin (1:10,000; Millipore) antibody and then with HRP-conjugated secondary antibody (1:8000; GE Healthcare, Little Chalfont, UK). The signals were detected by chemiluminescence and the density of each band was quantified by using the NIH Image J software.

Immunohistochemistry. The immunohistochemistry procedures were described in detail previously (Chao et al., 2006). The paraformaldehydefixed brains were cut into a series of $20 \mu \mathrm{m}$ coronal sections through the CA1 and dentate gyrus (DG) regions. Sections were incubated with mouse anti-HA antibody (1:100; Roche Products, Welwyn Garden City, $\mathrm{UK}$ ) and then with anti-mouse FITC-conjugated IgG (1:1000; Sigma, St. Louis, MO). Image acquisition was obtained by using a confocal microscope (Bio-Rad, Hercules, CA).
Water maze learning. The water maze used was a plastic, circular pool as described in detail previously (Lee et al., 2006). A circular platform was placed at a specific location away from the edge of the pool and was submerged $2.5 \mathrm{~cm}$ below the water surface. Because of transient expression of the plasmid DNA transfected (optimal expression between 48 and $72 \mathrm{~h}$ after transfection) (Abdallah et al., 1996), we have measured memory acquisition of the memory process. Behavioral procedures are described in detail in supplemental material (available at www.jneurosci.org).

Statistical analysis. Data for CK2 activity and Western blot were evaluated by Student's $t$ test or one-way ANOVA followed by the post hoc Newman-Keul's method. Water maze data were analyzed by two-way ANOVA with "group" as the independent measure and "block" as the repeated measure. Comparisons were made between groups across four blocks. Specific comparisons between each experimental group and control group were made with Newman-Keul's analysis after two-way ANOVA. $q$ values represent that calculated from separate sets of Newman-Keul's analysis.

\section{Results \\ CK2 activation negatively regulates spatial memory formation}

We first examined whether CK2 activation contributes to spatial memory formation. Naive rats were either subjected to water maze training (trained group) or swam for the same period of time without visual cues and platform (nontrained group). They were killed after training, and their hippocampal tissues containing the CA1 area and DG area were subjected to CK2 activity assay. Results revealed that water maze training significantly decreased CK2 activity in the CA1 area $\left(t_{(1,9)}=4.33 ; p=0.001\right)$; but, it only slightly decreased CK2 activity in the DG area $\left(t_{(1,9)}=\right.$ $1.09 ; p>0.05$ ) (Fig. 1A). These results suggest that CK2 activation in the CA1 area is negatively associated with spatial memory formation. We next transfected the dominant negative mutant of CK2, CK $2 \alpha \mathrm{A}^{156}$, to the CA1 and DG areas, respectively, and examined its effect on water maze performance. Results indicated that $\mathrm{CK} 2 \alpha \mathrm{A}^{156}$ transfection to the CA1 area significantly enhanced spatial memory formation $\left(F_{(1,14)}=5.48 ; p<0.05\right)$ (Fig. $1 B$, left); but, CK2 $\alpha \mathrm{A}^{156}$ transfection to the DG area did not markedly affect spatial memory $\left(F_{(1,10)}=0.17 ; p>0.05\right)$ (Fig. $1 B$, right). Immunohistochemistry staining against HA confirmed the transfection and expression of CK $2 \alpha \mathrm{A}^{156}$ in CA1 neurons and DG neurons (Fig. 1C). These results suggest that activation of CK2 in CA1 neurons impairs spatial memory formation.

\section{Inhibition of CK2 activates SGK1 and inactivates Akt}

We next examined the role of SGK1 and Akt possibly involved in the effect of CK2 on spatial memory formation. CK $2 \alpha \mathrm{A}^{156} \mathrm{mu}-$ tant plasmid was transfected to CA1 area and its effect on SGK1 and Akt phosphorylation was examined. Results revealed that CK2 $\alpha \mathrm{A}^{156}$ transfection significantly decreased CK2 activity in CA1 area for $\sim 38 \%$ (compared with control group; $t_{(1,10)}=4.22$, $p<0.01$ ), indicating that $\mathrm{CK} 2 \alpha \mathrm{A}^{156}$ transfection effectively downregulates CK2 signaling in CA1 neurons. Meanwhile, it markedly increased SGK1 phosphorylation at Ser422 $\left(t_{(1,10)}=\right.$ 3.83; $p<0.01$ ) (Fig. $1 D$, left) and decreased Akt phosphorylation at $\operatorname{Ser} 473\left(t_{(1,10)}=3.00 ; p<0.05\right)$ (Fig. $1 D$, middle). Because SGK1 was shown to directly phosphorylate cAMP response element-binding protein (CREB) in vitro (David and Kalb, 2005), we also examined the effect of CK $2 \alpha \mathrm{A}^{156}$ on CREB phosphorylation. Results revealed that $\mathrm{CK} 2 \alpha \mathrm{A}^{156}$ transfection to CA1 neurons markedly increased CREB Ser 133 phosphorylation $\left(t_{(1,10)}=\right.$ $2.21 ; p<0.05$ ) (Fig. $1 D$, right). 
(A)

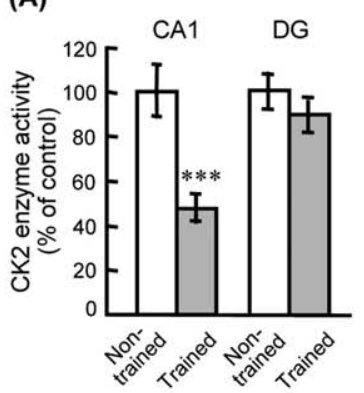

(B)
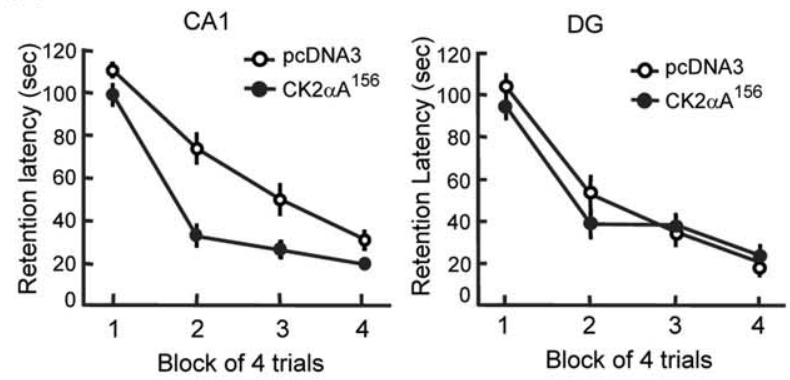

(C)
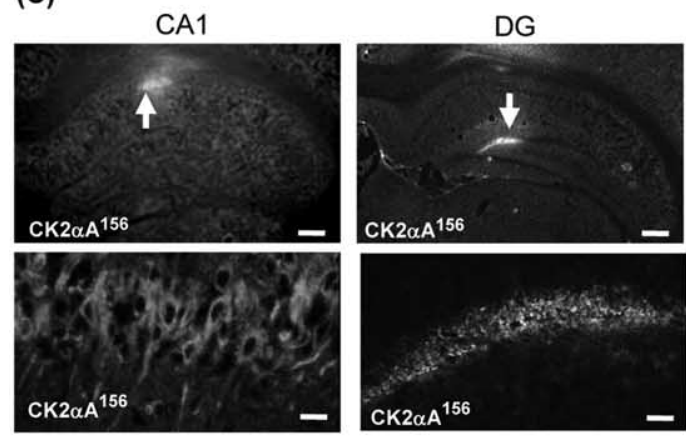

(D)
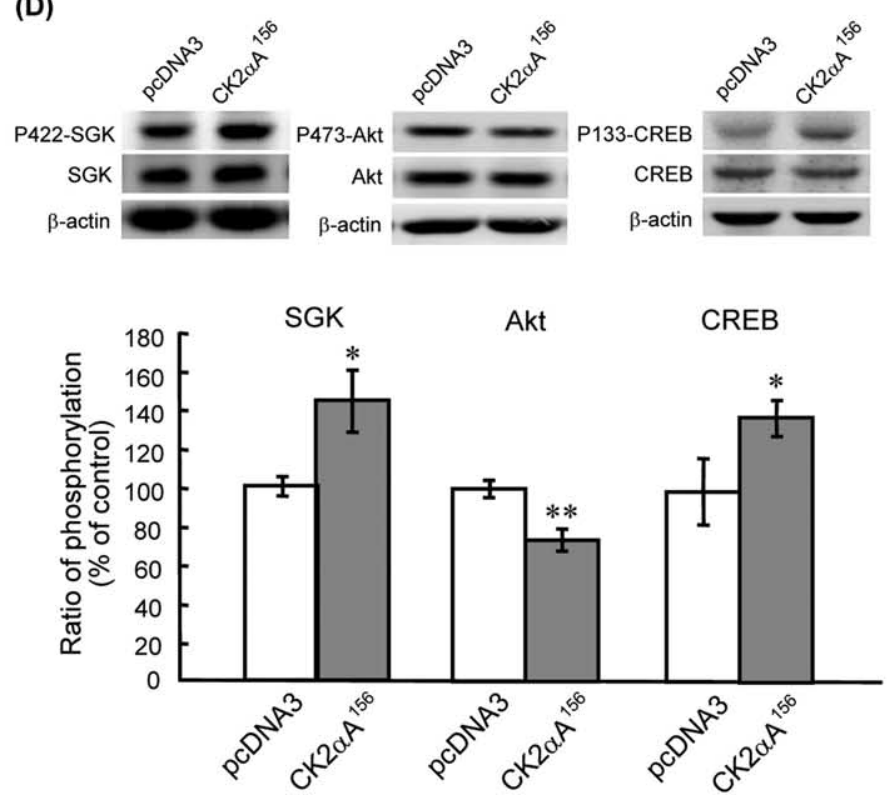

Figure 1. Effects of CK2 on spatial memory formation and phosphorylation of SGK and Akt. $A$, CK2 activity is markedly decreased in CA1 area, but not DG area, after water maze training ( $n=5$ each group). $B$, Overexpression of CK2 $\alpha A^{156}$ in CA1 neurons enhanced spatial memory formation (left; $n=7-8$ each group), but overexpression of CK2 $\alpha \mathrm{A}^{156}$ in DG neurons did not markedly affect spatial memory performance (right; $n=6$ each group). $C$, Immunohistochemistry showing the transfection and expression of $C K 2 \alpha \mathrm{A}^{156}$ in individual CA1 neurons and DG neurons. Arrow indicates the area of transfection. Scale bars: top, $400 \mu \mathrm{m}$; bottom $20 \mu \mathrm{m}$. D. Western blot showing that CK2 $\alpha \mathrm{A}^{156}$ transfection increased SGK1 phosphorylation at Ser422 (left), decreased Akt phosphorylation at Ser473 (middle), and increased CREB phosphorylation at Ser133 (right) in CA1 neurons. Data are mean \pm SEM. ${ }^{*} p<0.05 ;{ }^{* *} p<0.01 ;{ }^{* * *} p=0.001$.

\section{SGK1 enhances whereas Akt impairs spatial} memory formation

Because CK2 negatively regulates spatial memory formation and CK2 activates Akt and inactivates SGK1, we next examined the role of SGK1 and Akt involved in spatial memory formation. The dominant negative mutant of SGK1, SGKS422A, and the constitutively active SGK1, SGKS422D, were transfected to the CA1 area $48 \mathrm{~h}$ before water maze learning. Results revealed an overall significant effect of SGK1 plasmid transfection $\left(F_{(2,23)}=\right.$ $22.11 ; p<0.01$ ) (Fig. 2A). Further analyses indicated that SGKS422A markedly impaired $(q=4.32 ; p<0.01)$ whereas SGKS422D markedly enhanced $(q=2.85$; $p<0.05)$ spatial memory formation. Next, the wild-type (WT) Akt and the dominant negative mutant of Akt, AktS473A, were transfected to the CA1 area. Results revealed an overall significant effect of Akt plasmid transfection on water maze performance $\left(F_{(2,24)}=27.16\right.$; $p<0.01$ ) (Fig. $2 B$ ). Additional analyses indicated that AktWT impaired $(q=4.03$; $p<0.01$ ), whereas AktS473A facilitated $(q=3.3 ; p<0.01)$ spatial memory formation.

\section{CK2 inactivates SGK1 through PP2A}

CK2 was known to phosphorylate and activate Akt directly (Di Maira et al., 2005), but how CK2 regulates SGK activity is unknown. Because CK2 directly phosphorylates PP2A and upregulates its activity (Heriche et al., 1997), and PP2A inactivates SGK through dephosphorylation of SGK (Park et al., 1999), we therefore studied whether CK2 may regulate SGK1 activity through PP2A. CK2 $\alpha$ WT plasmid was first transfected to the CA1 area to increase CK2 enzyme activity ( $~ 84 \%$ when compared with the control group; $\left.t_{(1,13)}=3.72 ; p<0.01\right)$. Next, the PP2A inhibitor fostriecin (FST; $15 \mathrm{pg}$ ) was coadministered with $\mathrm{CK} 2 \alpha \mathrm{WT}$, and their effects on SGK1 phosphorylation was examined. This concentration of FST was adopted because our preliminary results indicated that $15 \mathrm{pg}$ FST did not significantly affect water maze performance (supplemental Fig. 1, available at www. jneurosci.org as supplemental material). Results revealed that $\mathrm{CK} 2 \alpha \mathrm{WT}$ transfection markedly decreased SGK1 phosphorylation at $\operatorname{Ser} 422\left(F_{(2,21)}=4.3, p<0.05\right.$; $q=3.75, p<0.05)$, whereas FST effectively reversed this effect of CK2 $2 \alpha \mathrm{WT}$ ( $q=$ 3.41; $p<0.05$ ) (Fig. 3A). Meanwhile, $\mathrm{CK} 2 \alpha \mathrm{WT}$ transfection moderately increased Akt phosphorylation at Ser473. Although this effect was further potentiated by FST, it did not reach a significant level $\left(F_{(2,21)}=1.82 ; p>0.05\right)$ (Fig. 3B). Figure $3 C$ is a representative illustration showing needle placement and dye distribution in the CA1 area.

CK2 impairs spatial memory formation through inactivation of SGK1 and activation of Akt

The above results showed that both CK2 and Akt negatively regulate spatial memory formation. CK2 also inactivated SGK1 through PP2A, whereas SGK1 facilitated spatial memory 
formation. We therefore examined whether CK2 may impair spatial memory through differential mediation by Akt and SGK1. Animals received pcDNA3+PBS, $\mathrm{CK} 2 \alpha \mathrm{WT}+\mathrm{PBS}, \mathrm{CK} 2 \alpha \mathrm{WT}+\mathrm{FST}$, and $\mathrm{CK} 2 \alpha \mathrm{WT}+\mathrm{AktS} 473 \mathrm{~A}$ transfections, respectively. Results revealed that $\mathrm{CK} 2 \alpha \mathrm{WT}$ markedly impaired spatial memory formation $\left(F_{(3,31)}=10.25, p<0.01 ; \mathrm{q}=\right.$ $5.47, p<0.05)$; but, this effect was antagonized by FST injection $(q=7.56$; $p<$ 0.01) (Fig. 3D), presumably because of SGK1 phosphorylation caused by PP2A inactivation. AktS473A transfection also reversed the memory-impairing effect of CK2 $\alpha$ WT $(q=3.61 ; p<0.05)$ (Fig. 3D). These results suggest that CK2 impairs spatial memory formation, at least partly, through the mediation of Akt.

\section{Discussion}

Previous studies have shown that CK2 activity is associated with the development of neuritogenesis (Ulloa et al., 1993) and the induction of LTP in hippocampal neurons (CharriautMarlangue et al., 1991). It is speculated that CK2 activation may also facilitate the learning and memory processes. However, our results showed that CK2 activity in CA1 neurons is negatively associated with spatial learning, and CK2 inactivation in CA1 neurons enhances spatial memory formation. We do not know the explanation for the discrepancy between our study and other studies yet. It could be possible that CK2 activation in different populations of neurons mediate different physiological functions.

In studying the signaling pathway involved in the memoryimpairing effect of CK2, we found that CK2 inactivation by CK $2 \alpha \mathrm{A}^{156}$ transfection increased SGK1 phosphorylation at Ser422 and decreased Akt phosphorylation at Ser473. In another study, SGK1 was shown to directly phosphorylate CREB (David and Kalb, 2005), and CREB plays an important role in memory formation (Silva et al., 1998). In assessing the possible downstream target of SGK1, we found that CK $2 \alpha \mathrm{A}^{156}$ transfection markedly increased CREB Ser133 phosphorylation. This result provides a rational explanation for the effect of CK $2 \alpha \mathrm{A}^{156}$ on SGK1 phosphorylation and memory facilitation. Whether Akt may also affect CREB activation is not known and awaits further investigation. Moreover, transfection of SGKS422D enhanced, whereas transfection of SGKS422A impaired spatial memory formation. These results are consistent with our previous finding that overexpression of SGK1WT facilitates spatial memory performance (Tsai et al., 2002). In another study, PI-3K activation was found to increase AMPA receptor insertion that is required for CA1 LTP (Man et al., 2003). Whether CK2 may affect AMPA receptor insertion to modulate memory processing awaits to be investigated. In further examination of the relationship between CK2 and SGK1, we found that CK2 inactivates SGK1 through activation of PP2A that directly dephosphorylates SGK1. These results are consistent with reports that $\mathrm{PP} 2 \mathrm{~A}$ acts as a negative regulator for ERK/MAPK (extracellular signal-regulated kinase/ mitogen-activated protein kinase) signaling (Kins et al., 2003; Grethe and Porn-Ares, 2006), because ERK/MAPK was shown to play an essential role in memory formation (Sweatt, 2004), and we have recently found that ERK/MAPK directly phosphorylates SGK1 (Lee et al., 2006). But these results are incongruent with the observations that PP2A inhibition blocks LTP induction in cerebellar neurons (Belmeguenai and Hansel, 2005) and that inhibition of PP1/PP2A impairs spatial memory formation (Sun et al., 2003). This is probably resulting from the unique characteristics of cerebellar LTP that depends on phosphatase rather than kinase activity. It is also likely caused by the difference in the concentration of PP2A inhibitors used. We have used $15 \mathrm{pg}$ (33 nM) of FST, but in other studies, $50 \mathrm{~nm}$ of FST application to cerebellar slice and $320 \mu \mathrm{M}$ calyculin A (CA) injection to hippocampus were used, whereas $100 \mu \mathrm{M}$ CA was shown to produce an Alzheimer's disease-like phosphatase deficiency system (Merrick et al., 1997). Although PP2A was found to also dephosphorylate and inactivate Akt (Ugi et al., 2004), which may diminish the effect of CK $2 \alpha$ on Akt activation, our results showed that the net effect of CK $2 \alpha$ activation is to increase Akt phosphorylation at Ser473 (Fig. 3D). In addition, earlier results showed that CK2 does not phosphorylate SGK directly (Di Maira et al., 2005), but our results cannot exclude the possibility that CK2 may inactivate SGK1 through molecules other than PP2A. For example, CK2 $\alpha$ was shown to phosphorylate and activate the MAP kinase phosphatase MKP3 (Castelli et al., 2004), and MKP3 was shown to dephosphorylate MAPK (Kim et al., 2003). Moreover, ERK/MAPK was found to directly phosphorylate SGK at Ser78 (Lee et al., 2006). Thus, CK2 may also inhibit SGK1 signaling through phosphorylation of MKP3 and inactivation of ERK/MAPK. Our results are also congruent with the report that overexpression of $\mathrm{CK} 2 \alpha$ results in deactivation of MEK (MAP kinase kinase) and negatively regulates ERK/MAPK signaling (Heriche et al., 1997).

In examination of the role of Akt involved in memory processing, we found that overexpression of AktWT impaired whereas transfection of AktS473A enhanced spatial memory formation. However, Horwood et al. (2006) have found that LTP induction rapidly and transiently increases Akt phosphorylation at Ser473 in hippocampal neurons. Meanwhile, Akt Ser473 phosphorylation was increased during recognition memory. In another study, Akt phosphorylation was increased after radial arm maze training (Mizuno et al., 2003). We do not know the explanation for the discrepancy between our study and these studies yet; however, in the LTP induction study, the authors have also found that although inhibition of PI-3K activity decreases Akt phosphorylation at Ser473, it does not affect spatial memory in rats. These latter results are indeed consistent with our findings because inhibition of PI-3K would inhibit the activity of both SGK1 and Akt, whereas the effect of SGK1 activation and Akt activation on spatial memory formation may compensate each other. Because 
(A)
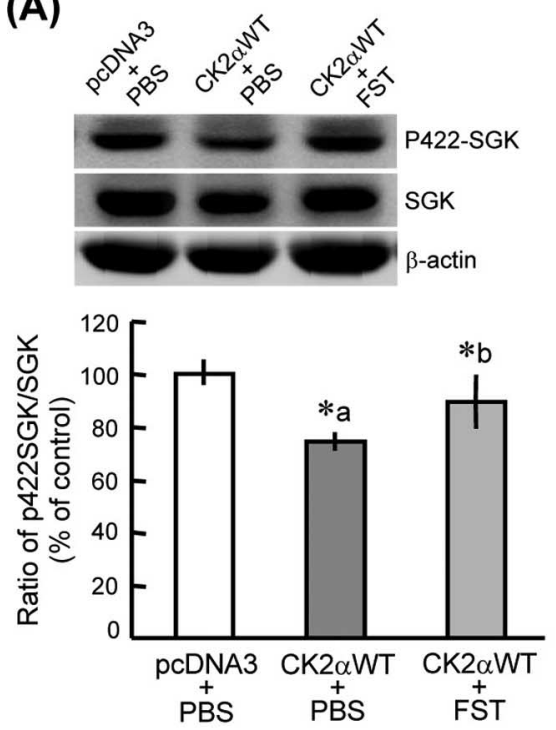

(B)
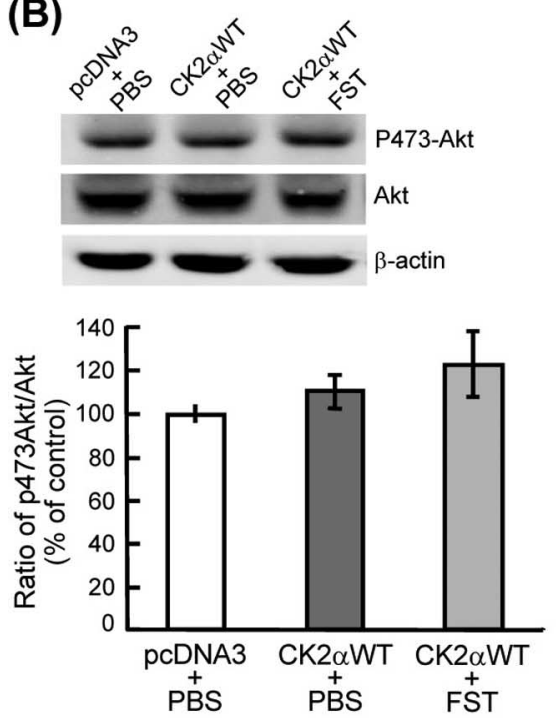

(C)

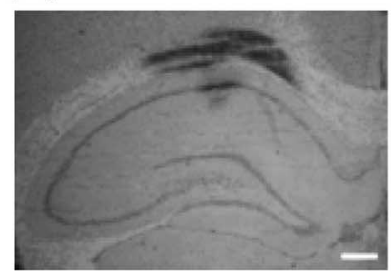

(D)

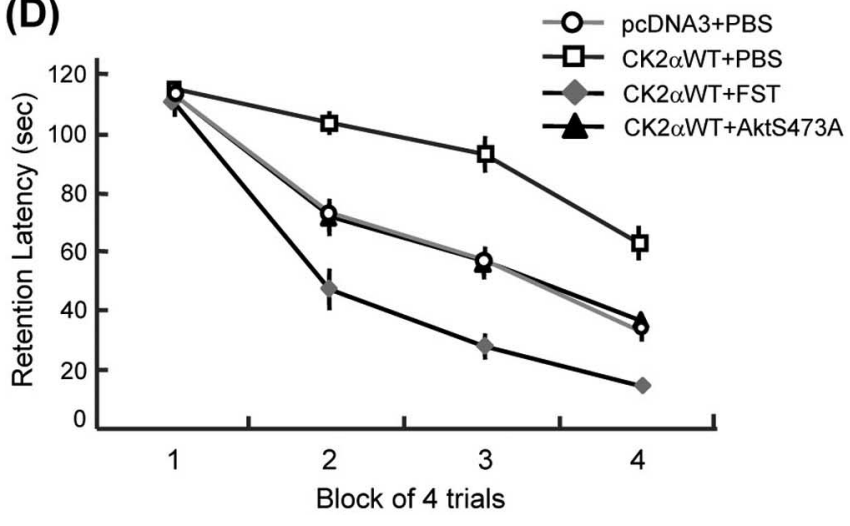

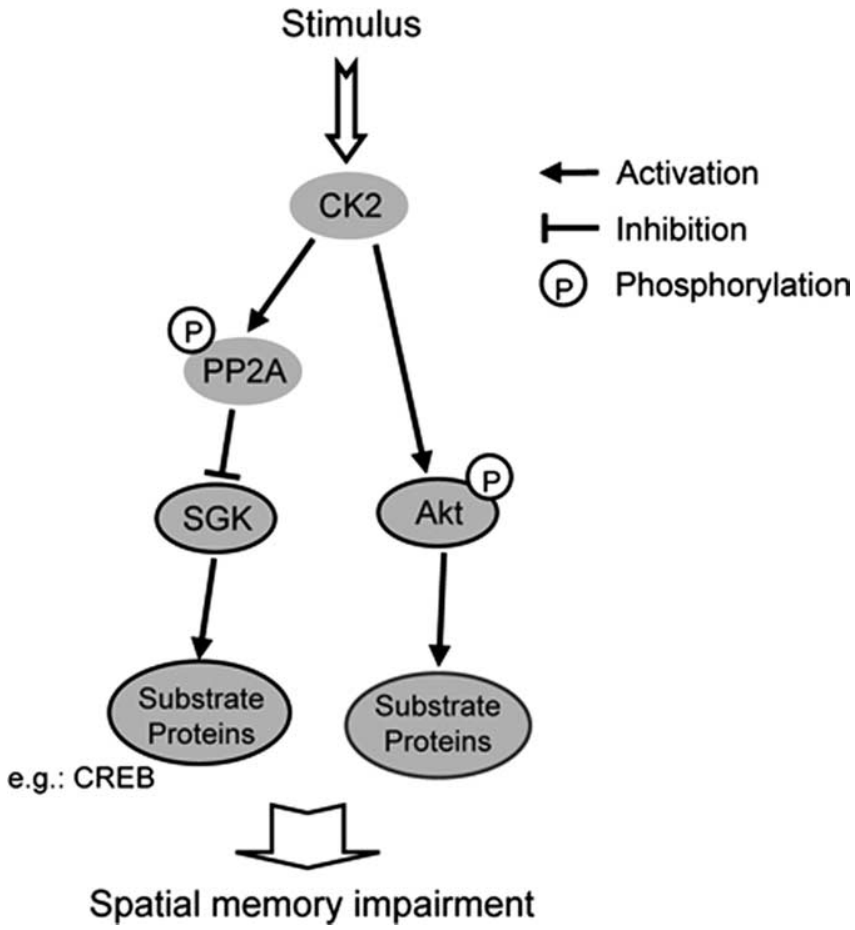

Figure 4. A schematic diagram showing the signaling cascade from CK2 to Akt and SGK1 involved in spatial memory formation. On stimulation, CK2 phosphorylates Akt directly, whereas overexpression of Akt impaired spatial memory formation through phosphorylation of substrate proteins. Conversely, CK2 activates PP2A, but PP2A inactivates SGK1 through dephosphorylation of SGK1. SGK1 facilitates spatial memory through phosphorylation of substrate proteins, such as CREB. Thus, CK2 impairs spatial memory formation through activation of Akt and inactivation of SGK1.

Akt phosphorylation was only transiently increased after LTP induction, it is possible that Akt is differentially activated during different stages of neuronal plasticity.

In an additional investigation of the relationship between CK2 and Akt, we found that Akt, at least in part, mediates the memory-impairing effect of CK $2 \alpha$. This result is consistent with another study showing that Akt mediates the anti-apoptotic effect of CK2 (Di Maira et al., 2005). Although Akt was phosphorylated directly by CK2 at Ser129, this would stimulate Akt autophosphorylation at Ser473 (Di Maira et al., 2005). In the present study, we found that CK2 impairs spatial memory formation through differential cross talk with SGK1 and Akt (Fig. 4), but the substrate proteins and downstream signaling of SGK1 and Akt that mediate the impairing effect of CK2 on memory performance awaits further investigation. Moreover, increasing evidence has shown that CK2 also plays a role in the maintenance of cell morphology and regulation of cytoskeleton proteins actin and tubulin (Canton and Litchfield, 2006). Because SGK1 was found to increase hippocampal neurite formation through mi-

\footnotetext{
Figure 3. Inhibition of PP2A and transfection of AktS473A both reversed the memoryimpairing effect of $\mathrm{CK} 2 \alpha$. A, CK2 $\alpha$ transfection decreased SGK1 phosphorylation at Ser422, but this effect is reversed by PP2A inhibition through FST administration ( $n=7-8$ each group). ${ }^{*} p<0.05$. A, Compared with control group; b, compared with CK2 $\alpha$ WT group. $\boldsymbol{B}$, CK2 $\alpha$ overexpression moderately increased Akt phosphorylation at Ser473, and this effect is slightly potentiated by FST injection. C, A representative illustration showing needle placement and dye distribution in the CA1 area. Scale bar, $400 \mu \mathrm{m}$. D, Both PP2A inhibition (by FST injection) and AktS473A transfection reversed the memory-impairing effect of CK2 $\alpha$ WT $(n=8-11$ each group). FST, Fostriecin. Data are mean \pm SEM. Both $p<0.01$.
} 
crotubule depolymerization (Yang et al., 2006), it is worth studying whether CK2 may inhibit neurite formation and alter cell morphology of hippocampal neurons through inactivation of SGK1.

\section{References}

Abdallah B, Hassan A, Benoist C, Goula D, Behr JP, Demeneix BA (1996) A powerful onviral vector for in vivo gene transfer into the adult mammalian brain: polyethylenimine. Hum Gene Ther 7:1947-1954.

Belmeguenai A, Hansel C (2005) A role for protein phosphatases 1, 2A, and 2B in cerebellar long-term potentiation. J Neurosci 25:10768-10772.

Blanquet PR (2002) Identification of two persistently activated neurotrophin-regulated pathways in rat hippocampus. J Neurosci 95:705-719.

Canton DA, Litchfield DW (2006) The shape of things to come: an emerging role for protein kinase CK2 in the regulation of cell morphology and the cytoskeleton. Cell Signal 18:267-275.

Castelli M, Camps M, Gillieron C, Leroy D, Arkinstall S, Rommel C, Nichols A (2004) MAP kinase phosphatase 3 (MKP3) interacts with and is phosphorylated by protein kinase CK2alpha. J Biol Chem 279:44731-44739.

Chao CC, Chiang CH, Lee EHY (2006) Molecular mechanism of the neuroprotective action of GDNF: role of protein kinase CK2. Neurobiol Aging 27:105-118.

Charriaut-Marlangue C, Otani S, Creuzet C, Ben-Ari Y, Loeb J (1991) Rapid activation of hippocampal casein kinase 2 during long-term potentiation. Proc Natl Acad Sci USA 88:10232-10236.

Chester N, Yu IJ, Marshak DR (1995) Identification and characterization of protein kinase CKII isoforms in HeLa cells. Isoform-specific differences in rates of assembly from catalytic and regulatory subunits. J Biol Chem 270:7501-7514.

David S, Kalb RG (2005) Serum/glucocorticoid-inducible kinase can phosphorylate the cyclic AMP response element binding protein, CREB. FEBS Lett 579:1534-1538.

Di Maira G, Salvi M, Arrigoni G, Marin O, Sarno S, Brustolon F, Pinna LA, Ruzzene M (2005) Protein kinase CK2 phosphorylates and upregulates Akt/PKB. Cell Death Differ 12:668-677.

Grethe S, Porn-Ares MI (2006) p38 MAPK regulates phosphorylation of Bad via PP2A-dependent suppression of the MEK1/2-ERK1/2 survival pathway in TNF-alpha induced endothelial apoptosis. Cell Signal $18: 531-540$

Heriche JK, Lebrin F, Rabilloud T, Leroy D, Chambaz EM, Goldberg Y (1997) Regulation of protein phosphatase 2A by direct interaction with casein kinase 2alpha. Science 276:952-955.

Horwood JM, Dufour F, Laroche S, Davis S (2006) Signalling mechanisms mediated by the phosphoinositide 3-kinase/Akt cascade in synaptic plasticity and memory in the rat. Eur J Neurosci 23:3375-3384.

Kim Y, Rice AE, Denu JM (2003) Intramolecular dephosphorylation of ERK by MKP3. Biochemistry 42:15197-15207.

Kins S, Kurosinski P, Nitsch RM, Gotz J (2003) Activation of the ERK and JNK signaling pathways caused by neuron-specific inhibition of PP2A in transgenic mice. Am J Pathol 163:833-843.

Kobayashi T, Cohen P (1999) Activation of serum- and glucocorticoidregulated protein kinase by agonists that activate phosphatidylinositide 3-kinase is mediated by 3-phosphoinositide-dependent protein kinase-1 (PDK1) and PDK2. Biochem J 339:319-328.

Lee CT, Tyan SW, Ma YL, Tsai MC, Yang YC, Lee EHY (2006) Serum- and glucocorticoid-inducible kinase (SGK) is a target of the MAPK/ERK signaling pathway that mediates memory formation in rats. Eur J Neurosci 23:1311-1320.

Lin CH, Yeh SH, Lin CH, Lu KT, Leu TH, Chang WC, Gean PW (2001) A role for the PI-3 kinase signaling pathway in fear conditioning and synaptic plasticity in the amygdala. Neuron 31:841-851.

Litchfield DW (2003) Protein kinase CK2: structure, regulation and role in cellular decisions of life and death. Biochem J 369:1-15.

Man HY, Wang Q, Lu WY, Ju W, Ahmadian G, Liu L, D’Souza S, Wong TP, Taghibiglou C, Lu J, Becker LE, Pei L, Liu F, Wymann MP, MacDonald JF, Wang YT (2003) Activation of PI3-kinase is required for AMPA receptor insertion during LTP of mEPSCs in cultured hippocampal neurons. Neuron 38:611-624.

Meggio F, Pinna LA (2003) One-thousand-and one-substrates of protein kinase CK2. FASEB J 17:349-368.

Merrick SE, Trojanowski JQ, Lee VM (1997) Selective destruction of stable microtubules and axons by inhibitors of protein serine/threonine phosphatases in cultured human neurons. J Neurosci 17:5726-5737.

Mizuno M, Yamada K, Takei N, Tran MH, He J, Nakajima A, Nawa H, Nabeshima T (2003) Phosphatidylinositol 3-kinase: a molecule mediating BDNF-dependent spatial memory formation. Mol Psychiatry 8:217-224.

Nakajo S, Hagiwara T, Nakaya K, Nakamura Y (1986) Tissue distribution of casein kinases. Biochem Int 13:701-707.

Park J, Leong ML, Buse P, Maiyar AC, Firestone GL, Hemmings BA (1999) Serum and glucocorticoid-inducible kinase (SGK) is a target of the PI3kinase-stimulated signaling pathway. EMBO J 18:3024-3033.

Pinna LA, Meggio F (1997) Protein kinase CK2 ("casein kinase-2") and its implication in cell division and proliferation. Prog Cell Cycle Res 3:77-97.

Silva AJ, Kogan JH, Frankland PW, Kida S (1998) CREB and memory. Annu Rev Neurosci 21:127-148.

Sun L, Liu SY, Zhou XW, Wang XC, Liu R, Wang Q, Wang JZ (2003) Inhibition of protein phosphatase $2 \mathrm{~A}$ - and protein phosphatase 1-induced tau hyperphosphorylation and impairment of spatial memory retention in rats. Neuroscience 118:1175-1182.

Sweatt JD (2004) Mitogen-activated protein kinases in synaptic plasticity and memory. Curr Opin Neurobiol 14:311-317.

Tsai KJ, Chen SK, Ma YL, Hsu WL, Lee EH (2002) Sgk, a primary glucocorticoid-induced gene, facilitates memory consolidation of spatial learning in rats. Proc Natl Acad Sci USA 99:3990-3995.

Ugi S, Imamura T, Maegawa H, Egawa K, Yoshizaki T, Shi K, Obata T, Ebina Y, Kashiwagi A, Olefsky JM (2004) Protein phosphatase 2A negatively regulates insulin's metabolic signaling pathway by inhibiting Akt (protein kinase B) activity in 3T3-L1 adipocytes. Mol Cell Biol 24:8778-8789.

Ulloa L, Diaz-Nido J, Avila J (1993) Depletion of casein kinase II by antisense oligonucleotide prevents neuritogenesis in neuroblastoma cells. EMBO J 12:1633-1640.

Yang YC, Lin CH, Lee EHY (2006) Serum- and glucocorticoid-inducible kinase 1 (SGK1) increases neurite formation through microtubule depolymerization by SGK1 and by SGK1 phosphorylation of tau. Mol Cell Biol 26:8357-8370. 